\title{
On the Cohomology of Some Complex Hyperbolic Arithmetic 3-Manifolds
}

\author{
Jian-Shu Li • Binyong Sun
}

Received: 15 August 2013 / Accepted: 21 September 2013 / Published online: 12 November 2013 (C) School of Mathematical Sciences, University of Science and Technology of China and Springer-Verlag Berlin Heidelberg 2013

\begin{abstract}
Given an arithmetic lattice of the unitary group $\mathrm{U}(3,1)$ arising from a hermitian form over a CM-field, we show that all unitary representations of $\mathrm{U}(3,1)$ with nonzero cohomology contribute to the cohomology of the attached arithmetic complex 3-manifold, at least when we pass to a finite-index subgroup of the given arithmetic lattice.
\end{abstract}

Keywords Cohomology · Unitary representation · Theta lift · Complex hyperbolic 3-manifold

Mathematics Subject Classification (2000) $11 \mathrm{~F} 70 \cdot 32 \mathrm{Q} 45 \cdot 55 \mathrm{~N} 25$

\section{Introduction}

Let $G$ be a connected reductive Lie group containing a compact Cartan subgroup. Let $K \subset G$ be a maximal compact subgroup and $X=G / K$ the global Riemannian symmetric space associated to $G$. Consider a lattice $\Gamma \subset G$. Let $F$ be a finite dimensional representation of $G$ and $\tilde{F}$ the local system on $\Gamma \backslash X$ determined by $F$.

The $L^{2}$-cohomology space $H_{(2)}^{*}(\Gamma \backslash X, \tilde{F})$ is defined to be the cohomology of the complex of $F$-valued smooth differential forms $\omega$ such that $\omega$ and $d \omega$ are both square integrable. (In particular, $H_{(2)}^{*}(\Gamma \backslash X, \tilde{F})$ agrees with the usual cohomology

\footnotetext{
J.-S. Li (凶)

Department of Mathematics, The Hong Kong University of Science Technology, Clear Water Bay, Hong Kong

e-mail: matom@ust.hk

B. Sun

Academy of Mathematics and Systems Science, Chinese Academy of Sciences, Beijing 100190, China

e-mail: sun@math.ac.cn
} 
$H^{*}(\Gamma \backslash X, \tilde{F})$ when $\Gamma \backslash G$ is compact.) This space is related to the decomposition of the regular representation of $G$ on $L^{2}(\Gamma \backslash G)$ via a Matsushima type formula as follows. Write $L^{2}(\Gamma \backslash G)$ as the direct sum of the discrete part $L_{d}^{2}(\Gamma \backslash G)$ and its orthogonal complement, the continuous part $L_{\text {eis }}^{2}(\Gamma \backslash G)$ furnished by Eisenstein series (see [17]). Write

$$
L_{d}^{2}(\Gamma \backslash G) \simeq \widehat{\bigoplus}_{\pi \in \hat{G}} m(\pi, \Gamma) H_{\pi},
$$

where $\hat{G}$ is the unitary dual of $G$ and the multiplicity $m(\pi, \Gamma)$ is a non-negative integer for each $\pi$. Since $G$ has a compact Cartan, the space $H_{(2)}^{*}(\Gamma \backslash X, \tilde{F})$ is finite dimensional and one has the formula [3]

$$
H_{(2)}^{*}(\Gamma \backslash X, \tilde{F}) \simeq \bigoplus_{\pi \in \hat{G}} m(\pi, \Gamma) H_{\mathrm{ct}}^{*}\left(G, H_{\pi} \otimes F\right),
$$

where $H_{\mathrm{ct}}^{*}$ denotes continuous cohomology. As already noted, $H_{(2)}^{*}(\Gamma \backslash X, \tilde{F})$ agrees with the ordinary cohomology space $H^{*}(\Gamma \backslash X, \tilde{F})$ when $\Gamma$ is cocompact, in which case (1.1) is the usual Matsushima formula, see [4, p. 223].

The representation $\pi$ with $H_{\mathrm{ct}}^{*}\left(G, H_{\pi} \otimes F\right) \neq 0$ turns out to be very special, and they have been completely classified by Vogan and Zuckerman [27]. Together with the formula (1.1), this implies a large amount of "automatic vanishing" for various parts of the $L^{2}$-cohomology. The purpose of this note is to show that, for $G=\mathrm{U}(3,1)$ and $\Gamma$ an arithmetic lattice arising from hermitian forms over CM-fields (see Sects. 4 and 5), these are essentially all the vanishing there is, at least when we pass to a finite-index subgroup of $\Gamma$.

Theorem 1.1 Let $G=\mathrm{U}(3,1)$ and $\Gamma$ an arithmetic lattice of $G$ arising from hermitian forms over CM-fields. Let $\pi$ be an irreducible unitary representation of $G$ with non-zero cohomology. Then there exists a subgroup $\Gamma^{\prime} \subseteq \Gamma$ of finite index, such that $m\left(\pi, \Gamma^{\prime}\right) \neq 0$. If $\pi$ is not one-dimensional then it occurs in the space of cusp forms.

It seems quite likely that the analogous result is valid for any $\mathrm{U}(n, 1)$. On the other hand, vanishing results with respect to congruence subgroups arising from division algebras with involutions of the second kind [5, 23, 24] indicate that the above restriction on the type of arithmetic subgroups is probably necessary.

\section{Unitary Representations with Non-zero Cohomology}

Let $G$ be a connected reductive Lie group containing a compact Cartan subgroup. We recall from [27, Sect. 5] the construction of irreducible unitary representations of $G$ with non-zero cohomology.

Let $\mathfrak{g}_{0}$ be the Lie algebra of $G$ with Cartan decomposition $\mathfrak{g}_{0}=\mathfrak{k}_{0}+\mathfrak{p}_{0}$. Let $\mathfrak{t}_{0} \subseteq \mathfrak{k}_{0}$ be a Cartan subalgebra of $\mathfrak{k}_{0}$ (and also for $\mathfrak{g}_{0}$ by our assumption). For $x \in i \mathfrak{t}_{0}$ the linear transformation $\operatorname{ad}(x)$ of $\mathfrak{g}$ is diagonalizable with real eigenvalues. Let $\mathfrak{q}$ (resp. $\mathfrak{u}$, resp. l) be the sum of non-negative (resp. positive, resp zero) eigenspaces of $\operatorname{ad}(x)$. 
Then $\mathfrak{q}$ is a parabolic subalgebra of $\mathfrak{g}$ with Levi decomposition $\mathfrak{q}=\mathfrak{l}+\mathfrak{u}$, and $\mathfrak{l}$ is the complexification of $\mathfrak{l}_{0}=\mathfrak{l} \cap \mathfrak{g}_{0}$. Let $K$ and $L$ be the connected subgroups of $G$ with Lie algebra $\mathfrak{k}_{0}$ and $\mathfrak{l}_{0}$, respectively. Let $\Delta(\mathfrak{g})$ denote the roots of $\mathfrak{t}$ in $\mathfrak{g}$. Fix a positive system $\Delta^{+}(\mathfrak{l})$ of the roots of $\mathfrak{t}$ in $\mathfrak{l}$. Then $\Delta^{+}(\mathfrak{g})=\Delta^{+}(\mathfrak{l}) \cup \Delta(\mathfrak{u})$ is a positive system for $\Delta(\mathfrak{g})$. Let $\rho$ be half the sum of roots in $\Delta^{+}(\mathfrak{t}, \mathfrak{g}), \rho(\mathfrak{u} \cap \mathfrak{p})$ half the sum of roots of $\mathfrak{t}$ in $\mathfrak{u} \cap \mathfrak{p}$. Let $\lambda: \mathfrak{l} \rightarrow \mathbb{C}$ be a one-dimensional representation of $\mathfrak{l}$ such that

$$
\left\{\begin{array}{l}
\text { (a) } \lambda \text { is the differential of a unitary character of } L ; \\
\text { (b) if } \alpha \in \Delta(\mathfrak{u}) \text {, then }\left\langle\alpha,\left.\lambda\right|_{\mathfrak{t}}\right\rangle \geq 0 .
\end{array}\right.
$$

Let $\mu(\mathfrak{q}, \lambda)$ be the representation of $K$ of highest weight $\left.\lambda\right|_{\mathfrak{t}}+2 \rho(\mathfrak{u} \cap \mathfrak{p})$. In [27, Theorem 5.3], Vogan and Zuckerman prove the existence of a unique irreducible unitary representation $A_{\mathfrak{q}}(\lambda)$ of $G$ with lowest $K$-type $\mu(\mathfrak{q}, \lambda)$ and infinitesimal character $\lambda+\rho$. In particular, the representation $A_{\mathfrak{q}}(\lambda)$ is in the discrete series if and only if $L$ is compact, and all discrete series representations of $G$ arise this way. Finally, every irreducible unitary representation with non-zero cohomology is of the form $A_{\mathfrak{q}}(\lambda)$.

Now we specialize to the case $G=\mathrm{U}(3,1)$. We realize $G$ as the group of linear transformations preserving the hermitian form on $\mathbb{C}^{4}$ with matrix $\operatorname{diag}(1,1,1,-1)$. We take $K=\mathrm{U}(3) \times \mathrm{U}(1)$, embedded in $G$ as block diagonal matrices. Let $\mathfrak{t}_{0}$ be the Cartan subalgebra consisting of diagonal matrices and identify $\sqrt{-1} t_{0}$ with $\mathbb{R}^{4}$ in the obvious manner. Since $G$ is of hermitian type, we have the usual decomposition $\mathfrak{p}=\mathfrak{p}^{+} \oplus \mathfrak{p}^{-}$into holomorphic and anti-holomorphic tangent spaces. The roots of $\mathfrak{t}$ in $\mathfrak{g}$ are $\Delta(\mathfrak{g})=\Delta(\mathfrak{k}) \cup \Delta\left(\mathfrak{p}^{+}\right) \cup \Delta\left(\mathfrak{p}^{-}\right)$, with

$$
\begin{gathered}
\Delta(\mathfrak{k})=\left\{ \pm\left(\varepsilon_{i}-\varepsilon_{j}\right) \mid 1 \leq i<j \leq 3\right\}, \\
-\Delta\left(\mathfrak{p}^{-}\right)=\Delta\left(\mathfrak{p}^{+}\right)=\left\{\left(\varepsilon_{i}-\varepsilon_{4}\right) \mid i=1,2,3\right\},
\end{gathered}
$$

where $\varepsilon_{i}$ denotes evaluation on the $i$ th coordinate.

Take

$$
x=\left(x_{1}, x_{2}, x_{3} ; x_{4}\right) \in \sqrt{-1} t_{0}
$$

and consider the corresponding parabolic subalgebra $\mathfrak{q}$ as in the first paragraph of this page. Up to conjugation by $K$, we may assume $x_{1} \geq x_{2} \geq x_{3}$. The following lemma is obvious.

Lemma 2.1 Assume that $x$ is not in the center of $\mathfrak{g}$, i.e., its coordinates are not all equal.

(a) If $x_{3} \geq x_{4}$ then $\Delta(\mathfrak{u}) \subseteq \Delta\left(\mathfrak{p}^{+}\right)$, and the corresponding $A_{\mathfrak{q}}(\lambda)$ 's are holomorphic (lowest weight) representations. Similarly if $x_{4} \geq x_{1}$ then the corresponding $A_{\mathfrak{q}}(\lambda)$ 's are anti-holomorphic representations.

(b) Assume $x_{i} \neq x_{4}$ for $i=1,2,3$. Then $L$ is compact, and the corresponding $A_{\mathfrak{q}}(\lambda)$ 's are discrete series representations.

Excluding the situations considered in the preceding lemma, we have

$$
x=\left(x_{1}, x_{2}, x_{3} ; x_{2}\right), \quad x_{1}>x_{2}>x_{3} .
$$


All such $x$ 's define the same q. So from now on we fix one of them, say

$$
x=x_{0}=(1,0,-1 ; 0)
$$

and let $\mathfrak{q}=\mathfrak{l} \oplus \mathfrak{u}$ be the parabolic subalgebra defined by $x_{0}$. This is the case of interest to us. Note that

$$
L \simeq \mathrm{U}(1)^{2} \times \mathrm{U}(1,1), \quad \Delta(\mathfrak{u} \cap \mathfrak{p})=\left\{\varepsilon_{1}-\varepsilon_{4},-\varepsilon_{3}+\varepsilon_{4}\right\} .
$$

Since $G$ is of hermitian type, for any $(\mathfrak{g}, K)$-module $V$ we have a natural decomposition of cohomology spaces

$$
H^{n}(\mathfrak{g}, \mathfrak{k}, V)=\bigoplus_{p+q=n} H^{p, q}(\mathfrak{g}, \mathfrak{k}, V),
$$

corresponding to the Hodge structure on the cohomology of locally symmetric spaces via the Matsushima formula (1.1). By Proposition 6.19 of [27] we easily obtain the following result. Here we will abuse notations slightly to write $A_{\mathfrak{q}}(\lambda)$ also for the Harish-Chandra module underlying the unitary representation.

Proposition 2.2 Let $\mathfrak{q}$ be defined by the element $x_{0}$ of (2.2), and let $\lambda$ be a onedimensional representation of $\mathfrak{l}$ satisfying (2.1). Let $F$ be a finite dimensional irreducible representation of $G$.

(a) If the lowest weight of $F$ is $-\left.\lambda\right|_{\mathfrak{t}}$ then

$$
\operatorname{dim} H^{1,1}\left(\mathfrak{q}, \mathfrak{k}, A_{\mathfrak{q}}(\lambda) \otimes F\right)=\operatorname{dim} H^{2,2}\left(\mathfrak{q}, \mathfrak{k}, A_{\mathfrak{q}}(\lambda) \otimes F\right)=1
$$

and $H^{p, q}\left(\mathfrak{q}, \mathfrak{k}, A_{\mathfrak{q}}(\lambda) \otimes F\right)=0$ whenever $(p, q) \neq(1,1)$ or $(2,2)$.

(b) If the lowest weight of $F$ is not $-\left.\lambda\right|_{\mathfrak{t}}$ then $H^{i}\left(\mathfrak{q}, \mathfrak{k}, A_{\mathfrak{q}}(\lambda) \otimes F\right)=0$ for all $i$.

Since $\lambda$ is one-dimensional, it is determined by its restriction to $t$. We will therefore abuse notations slightly to denote $\left.\lambda\right|_{\mathfrak{t}}$ by $\lambda$. Then, any $\lambda$ satisfying (2.1) is of the form

$$
\lambda=\lambda_{r}=(a+r, r,-b+r ; r) \quad(a, b, r \in \mathbb{Z}, a, b \geq 0) .
$$

In particular,

$$
\lambda_{0}=(a, 0,-b ; 0) \quad(a, b \in \mathbb{Z}, a, b \geq 0) .
$$

Then clearly

$$
A_{\mathfrak{q}}(\lambda)=A_{\mathfrak{q}}\left(\lambda_{r}\right) \simeq A_{\mathfrak{q}}\left(\lambda_{0}\right) \otimes \operatorname{det}^{r},
$$

where det denotes the determinant character of $U(3,1)$.

Consider now another unitary group $\mathrm{U}(1,1)$. As is well known, $\mathrm{U}(3,1)$ and $\mathrm{U}(1,1)$ together form an example of what is called a reductive dual pair inside the symplectic group $\operatorname{Sp}_{16}(\mathbb{R})$. Let $\omega$ be the smooth oscillator representation of metaplectic cover of $\operatorname{Sp}_{16}(\mathbb{R})$ attached to the additive character $t \mapsto \mathrm{e}^{i a x}$, with some fixed $a>0$. (For all this, see [10].) When restricted to the subgroups, $\omega$ can be considered 
an ordinary representation of the product $U(3,1) \times U(1,1)$. More precisely, the splitting of the metaplectic cover over $\mathrm{U}(3,1) \times \mathrm{U}(1,1)$ depends on the choice of a pair of characters $\chi=\left(\chi_{1}, \chi_{2}\right)$ of $\mathbb{C}^{\times}$with trivial restrictions to $\mathbb{R}^{\times}$(cf. [14]). With this in mind, we speak of $\chi$-theta liftings. We could in particular choose both $\chi_{1}$ and $\chi_{2}$ to be trivial, in such case we simply use the term "theta lifting".

We recall the following result from [19], specialized to the present case.

Proposition 2.3 The representation $A_{\mathfrak{q}}\left(\lambda_{0}\right)$ in (2.5) is the local theta lift from the holomorphic discrete series representation $\pi^{\prime}=\pi^{\prime}(a, b)$ of $\mathrm{U}(1,1)$ with lowest weight $(a+2,-b-2)$.

Remark 2.4 As explained above the theta lift in the proposition uses the trivial splitting character. If instead we choose $\chi_{2}$ to be trivial but let

$$
\chi_{1}(c)=\left(\begin{array}{l}
c \\
\bar{c}
\end{array}\right)^{r} \quad\left(c \in \mathbb{C}^{\times}\right)
$$

with $r$ as in (2.3) and write $\chi_{r}=\left(\chi_{1}, 1\right)$ for the resulting pair, then $A_{\mathfrak{q}}(\lambda)$ is the $\chi_{r}$-theta lift of $\pi^{\prime}(a, b)$.

It is important to note that the theta lifting in the above proposition is explicitly constructed (in [19]) as follows. Let $\pi^{\prime \vee}$ be the contragredient of $\pi^{\prime}=\pi^{\prime}(a, b)$, and suppose it is realized on some Hilbert space $\mathcal{V}$. Let $\mathcal{V}^{\infty} \subset \mathcal{V}$ be the subspace of smooth vectors. Similarly let $\mathcal{W}$ be the space for the smooth oscillator representation $\omega$. On the algebraic tensor product $\mathcal{W} \otimes \mathcal{V}^{\infty}$ we have the actions of $U(3,1)$ and $\mathrm{U}(1,1)$, where $\mathrm{U}(3,1)$ acts on the first factor via $\omega$, and $\mathrm{U}(1,1)$ acts by $\omega \otimes \pi^{\prime \vee}$. Consider the integrals

$$
\int_{\mathrm{U}(1,1)}\left\langle\omega \otimes \pi^{\prime \vee}(h) u, v\right\rangle d h \quad\left(u, v \in \mathcal{W} \otimes \mathcal{V}^{\infty}\right),
$$

where $d h$ denotes a fixed Haar measure on $\mathrm{U}(1,1)$. These integrals are absolutely convergent, and the resulting sesquilinear form on $\mathcal{W} \otimes V^{\infty}$ is positive semi-definite. Let $R$ be the radical of this sesquilinear form. Then $\mathcal{W} \otimes \mathcal{V}^{\infty} / R$ is a pre-Hilbert space on which $G=\mathrm{U}(3,1)$ acts unitarily, and the resulting unitary representation on the completed Hilbert space is precisely $A_{\mathfrak{q}}\left(\lambda_{0}\right)$.

For later use we also record the known theta correspondence for the dual pairs $\mathrm{U}(4), \mathrm{U}(2)$ and $\mathrm{U}(4), \mathrm{U}(1,1)$, also with respect to the trivial splitting character.

Proposition 2.5 [12] (a) The theta correspondence for the compact dual pair U(4), $\mathrm{U}(2)$ is given as follows. An irreducible representation of $\mathrm{U}(2)$ occurs in the correspondence if and only if its highest weight is of the form $(a+2, b+2)$ with $a \geq b \geq 0$. In such case the corresponding representation of $\mathrm{U}(4)$ is of highest weight $(a+1, b+1,1,1)$.

(b) An irreducible representation of $\mathrm{U}(1,1)$ occurs in theta correspondence with $\mathrm{U}(4)$ if and only it is a holomorphic discrete series representation with lowest weight $(a+2,-b-2)$, where $a, b \geq 0$. In such case the corresponding representation of $\mathrm{U}(4)$ is of highest weight $(a, 0,0,-b)$. 


\section{Non-vanishing of Certain Integrals of Matrix Coefficients}

In this section only, assume that $F$ is a non-Archimedean local field of characteristic zero. Let $E$ be a separable $F$-algebra of dimension 2. In other words, $E$ is either a quadratic field extension of $F$, or a product of two copies of $F$. Let $V$ be a hermitian $E$-module, namely, it is a free $E$-module of finite rank, equipped with a non-degenerate $F$-bilinear map

$$
\langle,\rangle: V \times V \rightarrow E
$$

such that

$$
\langle u, v\rangle=\langle v, u\rangle^{\iota}, \quad\langle a u, v\rangle=a\langle u, v\rangle, \quad a \in E, u, v \in V .
$$

Here " $\iota$ " denotes the non-trivial automorphism of $E$ (as an $F$-algebra). Likewise, let $V^{\prime}$ be a skew hermitian $E$-module. Then

$$
W:=V \otimes_{E} V^{\prime}
$$

is a symplectic space over $F$ under the form

$$
\left\langle u \otimes u^{\prime}, v \otimes v^{\prime}\right\rangle:=\operatorname{tr}_{E / F}\left(\langle u, v\rangle \cdot\left\langle u^{\prime}, v^{\prime}\right\rangle\right) .
$$

Denote by

$$
\mathrm{H}(W):=W \times F
$$

the associated Heisenberg group, whose multiplication is given by

$$
(w, t)\left(w^{\prime}, t^{\prime}\right):=\left(w+w^{\prime}, t+t^{\prime}+\left\langle w, w^{\prime}\right\rangle\right) .
$$

The product $\mathrm{U}(V) \times \mathrm{U}\left(V^{\prime}\right)$ of unitary groups (or general linear groups in the split case) acts on $\mathrm{H}(W)$ as group automorphisms through its natural action on $W$. We define the associated Jacobi group to be the semi-direct product

$$
\mathrm{J}:=\left(\mathrm{U}(V) \times \mathrm{U}\left(V^{\prime}\right)\right) \ltimes \mathrm{H}(W) .
$$

Fix a non-trivial unitary character $\psi: F \rightarrow \mathbb{C}^{\times}$. Assume that $V$ and $V^{\prime}$ have respective ranks 4 and 2 , as free $E$-modules. Write $\omega$ for the smooth oscillator representation of $\mathbf{J}$ attached to $\psi$ and the trivial splitting characters, that is, up to isomorphism, it is the unique smooth representation of $\mathrm{J}$ with the following properties:

- as a representation of $\mathrm{H}(W)$, it is irreducible with central character $\psi$;

- both the representation $\left.\omega\right|_{\mathrm{U}(V) \ltimes \mathrm{H}(W)}$ and $\left.\omega\right|_{\mathrm{U}\left(V^{\prime}\right) \ltimes \mathrm{H}(W)}$ have trivial Kudla characters (see [9, Sect. B.2] for the notion of Kudla characters).

Note that the contragredient $\omega^{\vee}$ of $\omega$ is isomorphic to the smooth oscillator representation of $\mathbf{J}$ attached to $\psi^{-1}$ and the trivial splitting characters.

Let $\pi^{\prime}$ be a unitarizable irreducible smooth representation of $U\left(V^{\prime}\right)$, and write $\pi^{\prime \vee}$ for its contragredient. 
Proposition 3.1 The integrals in

$$
\begin{aligned}
\omega \times \pi^{\prime \vee} \times \omega^{\vee} \times \pi^{\prime} & \rightarrow \mathbb{C}, \\
\left(\phi, v^{\vee}, \phi^{\vee}, v\right) & \mapsto \int_{\mathrm{U}\left(V^{\prime}\right)}\left\langle g^{\prime} \cdot \phi, \phi^{\vee}\right\rangle\left\langle g^{\prime} \cdot v^{\vee}, v\right\rangle d g^{\prime},
\end{aligned}
$$

are absolutely convergent, where $d g^{\prime}$ denotes a Haar measure on $\mathrm{U}\left(V^{\prime}\right)$. The map (3.1) is not identically zero if $E$ splits, or $V$ splits, or $\pi^{\prime}$ is not the trivial representation.

Proof Write $V^{\prime-}$ for the space $V^{\prime}$ equipped with the form scaled by -1 . Put

$$
V^{\prime \square}:=V^{\prime} \oplus V^{\prime-} \text {. }
$$

It has a Lagrangian submodule

$$
V^{\prime \Delta}:=\left\{\left(v^{\prime}, v^{\prime}\right) \in V^{\prime} \square .\right.
$$

As before,

$$
W^{\square}:=V \otimes_{E} V^{\prime \square}
$$

is a symplectic space over $F$. It has a Lagrangian subspace

$$
W^{\Delta}:=V \otimes_{E} V^{\prime \Delta}
$$

and an orthogonal decomposition

$$
W^{\square}:=W \oplus W^{-}, \quad \text { where } W^{-}:=V \otimes_{E} V^{\prime-} .
$$

Denote by $\omega^{\square}$ the smooth oscillator representation of

$$
\mathrm{J}^{\square}:=\left(\mathrm{U}(V) \times \mathrm{U}\left(V^{\prime}\right)\right) \ltimes \mathrm{H}\left(W^{\square}\right),
$$

attached to $\psi$ and the trivial splitting characters. Fix a non-zero linear functional $\lambda_{\Delta}$ on $\omega^{\square}$ which is invariant under

$$
W^{\Delta} \subset W^{\square} \subset \mathrm{H}\left(W^{\square}\right) \subset \mathrm{J}^{\square} .
$$

It is unique up to scalar multiplications. Moreover, for all $p$ in the parabolic subgroup $\mathrm{P}\left(V^{\prime \Delta}\right)$ of $\mathrm{U}\left(V^{\square}\right)$ stabilizing $V^{\prime \Delta}$, one has (cf. [9, Sect. B.2])

$$
\lambda_{\Delta}\left(p \phi^{\square}\right)=|p|^{2} \lambda_{\Delta}\left(\phi^{\square}\right), \quad \phi^{\square} \in \omega^{\square} .
$$

Here $|p|$ denotes the value at $p$ of the following positive character:

$$
\mathrm{P}\left(V^{\prime \Delta}\right) \stackrel{\text { restriction on } V^{\prime \Delta}}{\longrightarrow} \mathrm{GL}\left(V^{\prime \Delta}\right) \stackrel{\text { det }}{\longrightarrow} E^{\times} \stackrel{\text { normalized absolute value }}{\longrightarrow} \mathbb{R}_{+}^{\times} .
$$

Write

$$
\eta: \omega \otimes \omega^{\vee} \stackrel{\sim}{\rightarrow} \omega^{\square}
$$

for the unique linear isomorphism with the following two properties: 
- it is equivariant with respect to the homomorphism

$$
\mathrm{H}(W) \times \mathrm{H}(W) \stackrel{1 \times \varepsilon_{W}}{\longrightarrow} \mathrm{H}(W) \times \mathrm{H}\left(W^{-}\right) \stackrel{\varsigma}{\rightarrow} \mathrm{H}\left(W^{\square}\right),
$$

where $\varepsilon_{W}$ denotes the isomorphism $\mathrm{H}(W) \rightarrow \mathrm{H}\left(W^{-}\right),(w, t) \mapsto(w,-t)$ (note that $W=W^{-}$as vector spaces over $F$ ), and $\varsigma$ denotes the homomorphism $\left(w, t ; w^{-}, t^{\prime}\right) \mapsto\left(w, w^{-} ; t+t^{\prime}\right)$;

- $\lambda_{\Delta} \circ \eta$ coincides with the canonical paring between $\omega$ and $\omega^{\vee}$.

Then $\eta$ is also equivariant with respect to the inclusion (cf. [26, Sect. 3])

$$
\mathrm{U}\left(V^{\prime}\right) \times \mathrm{U}\left(V^{\prime}\right)=\mathrm{U}\left(V^{\prime}\right) \times \mathrm{U}\left(V^{\prime-}\right) \subset \mathrm{U}\left(V^{\prime} \square\right) .
$$

Consequently, the following diagram commutes:

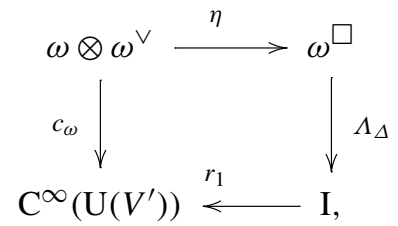

where

$$
\mathrm{I}:=\left\{\left.f \in \mathrm{C}^{\infty}\left(\mathrm{U}\left(V^{\prime \square}\right)\right)|f(p x)=| p\right|^{2} f(x), p \in \mathrm{P}\left(V^{\prime \Delta}\right), x \in \mathrm{U}\left(V^{\prime}\right)\right\}
$$

is a representation of $\mathrm{U}\left(V^{\prime} \square\right)$ under right translations; $\Lambda_{\Delta}$ denotes the map

$$
\phi^{\square} \mapsto\left(g \mapsto \lambda_{\Delta}\left(g \phi^{\square}\right)\right) ;
$$

$c_{\omega}$ denotes the matrix coefficient map

$$
\phi \otimes \phi^{\vee} \mapsto\left(g \mapsto\left\langle g . \phi, \phi^{\vee}\right\rangle\right)
$$

and $r_{1}$ denotes the restriction through the inclusion $\mathrm{U}\left(V^{\prime}\right) \subset \mathrm{U}\left(V^{\prime} \square\right)$.

Note that there is a commutative diagram

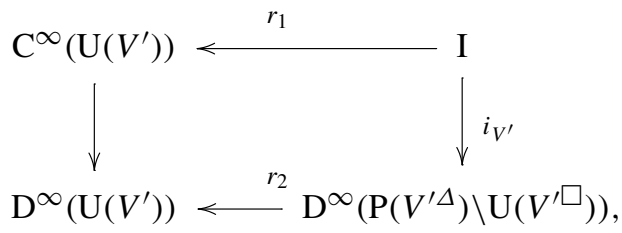

where " $\mathrm{D}^{\infty}$ " stands for the space of smooth measures; $i_{V^{\prime}}$ is a $\mathrm{U}\left(V^{\prime} \square\right)$-equivariant linear isomorphism; $r_{2}$ is the restriction map through the open embedding $\mathrm{U}\left(V^{\prime}\right) \hookrightarrow$ $\mathrm{P}\left(V^{\prime \Delta}\right) \backslash \mathrm{U}\left(V^{\prime} \square\right)$; and the left vertical arrow is the linear isomorphism of multiplying by the Haar measure $d g^{\prime}$. 
Since $\mathrm{P}\left(V^{\prime \Delta}\right) \backslash \mathrm{U}\left(V^{\prime} \square\right)$ is compact, every element in the image of $r_{2}$ is integrable. Therefore the image of $c_{\omega}$ consists of only integrable functions. This proves the first assertion of the proposition.

If either $E$ or $V$ splits, then by [15, Theorem 1.2], the map $\Lambda_{\Delta}$ is surjective. Therefore, the image of $c_{\omega}$ contains the space of all Schwartz functions on $\mathrm{U}\left(V^{\prime}\right)$, and the proposition follows.

Now assume that $E$ is a field, and $V$ does not split. Then [15, Theorem 1.2] implies that the image of $i_{V^{\prime}} \circ \Lambda_{\Delta}$ equals the space of all smooth measures on $\mathrm{P}\left(V^{\prime \Delta}\right) \backslash \mathrm{U}\left(V^{\prime} \square\right)$ whose integrals vanish. Therefore, the image of $c_{\omega}$ contains the space of all Schwartz functions on $\mathrm{U}\left(V^{\prime}\right)$ whose integrals vanish. If $\pi^{\prime}$ is not the trivial representation, then $\pi^{\prime}$ has a bounded non-constant matrix coefficient, and the proposition follows easily.

We remark that when $V$ splits, the dual pair $\mathrm{U}(V), \mathrm{U}\left(V^{\prime}\right)$ is in the so called stable range, and the assertion of the proposition also follows from [18].

\section{Global Theta Lifting}

From now on let $F$ be a totally real number field, and $E / F$ a totally imaginary quadratic extension of $F$. Let $V$ be a hermitian space over $E$ of dimension 4 . We assume that

$$
\left\{\begin{array}{l}
\text { (a) for one fixed real place } v_{0}, V \text { has signature }(3,1) ; \\
\text { (b) at all other real places, } V \text { is positive definite. }
\end{array}\right.
$$

View the isometry group $\mathbb{G}=\mathrm{U}(V)$ as an algebraic group over $F$. By the above assumption, we see that $\mathbb{G}\left(F_{v}\right)=\mathrm{U}(3,1)$ for $v=v_{0}$, and $\mathbb{G}\left(F_{v}\right)=\mathrm{U}(4)$ for any other real place $v$.

Let $V^{\prime}$ be a skew-hermitian space over $E$ of dimension 2. Let $\mathbb{G}^{\prime}=\mathrm{U}\left(V^{\prime}\right)$ be the corresponding unitary group. Then for any real place $v, \mathbb{G}^{\prime}\left(F_{v}\right)$ is isomorphic to either $\mathrm{U}(2)$ or $\mathrm{U}(1,1)$. We assume that $\mathbb{G}^{\prime}\left(F_{v_{0}}\right)$ is isomorphic to $\mathrm{U}(1,1)$, i.e., $V^{\prime}$ splits at the place $v_{0}$.

Set

$$
W=V \otimes_{E} V^{\prime}
$$

As in the preceding section, this is a symplectic space over $F$ of dimension 16 . Let $\mathrm{Sp}(W)$ be the corresponding symplectic group. We have a natural map

$$
\iota: \mathbb{G} \times \mathbb{G}^{\prime} \longrightarrow \mathrm{Sp}(W)
$$

given by the obvious actions of $\mathbb{G}$ and $\mathbb{G}^{\prime}$ on $W$. We remark that the reductive dual pair $\mathbb{G}, \mathbb{G}^{\prime}$ is outside the range considered in [20].

Let $\mathbb{A}=\mathbb{A}_{F}$ be the ring of adeles of $F$. Fix a non-trivial character $\psi=\prod \psi_{v}$ of $\mathbb{A} / F$. We assume that for each real place $v$ of $F$, there is a positive element $a \in F_{v} \simeq \mathbb{R}$, such that

$$
\psi_{v}(x)=\mathrm{e}^{i a x} \quad\left(x \in F_{v}\right)
$$


Let $\omega_{\psi}$ be the oscillator representation of the metaplectic group $\operatorname{Mp}(W)$ attached to $\psi$. We recall that $\operatorname{Mp}(W)$ fits into the short exact sequence

$$
1 \longrightarrow S^{1} \longrightarrow \mathrm{Mp}(W) \longrightarrow \mathrm{Sp}(W)_{\mathbb{A}} \longrightarrow 1 \text {. }
$$

Let $C_{F}$ and $C_{E}$ be the idele class groups of $F$ and $E$, respectively. Recall from [14] and [8] that for any pair of characters $\chi=\left(\chi_{1}, \chi_{2}\right)$ of $C_{E}$ satisfying certain condition-which means trivial restriction to $C_{F}$ in the present context-we have a homomorphism

$$
\iota_{\chi}: \mathbb{G}(\mathbb{A}) \times \mathbb{G}^{\prime}(\mathbb{A}) \longrightarrow \operatorname{Mp}(W)
$$

lifting $\iota$. In other words, we have the commutative diagram

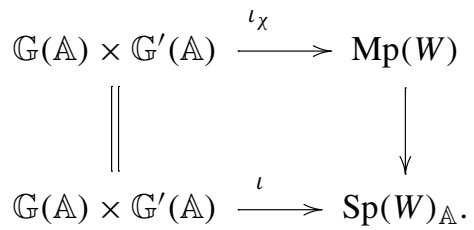

Let $\omega_{\chi}=\omega_{\chi, \psi}$ be the composition of $\iota_{\chi}$ with $\omega_{\psi}$. In this paper, we shall just take $\chi$ to be trivial (i.e., both $\chi_{1}$ and $\chi_{2}$ are trivial). We write 1 for this trivial $\chi$, and let $\omega=\omega_{1}=\omega_{1, \psi}$. This is a restricted tensor product: $\omega=\otimes \omega_{v}$, where each $\omega_{v}$ is a local oscillator representation as discussed in Sects. 2 and 3. As is well known, the representation $\omega$ may be realized on the Schwartz space $S(X(\mathbb{A}))$, where $X$ is any Lagrange subspace of the symplectic space $W$. The theta-distribution is defined by

$$
\theta(\phi)=\sum_{\xi \in X(k)} \phi(\xi) \quad(\phi \in S(X(\mathbb{A})))
$$

which satisfies

$$
\theta(\omega(\gamma) \phi)=\theta(\phi) \quad\left(\gamma \in \operatorname{Sp}(W)_{F}\right) .
$$

For any fixed Schwartz function $\phi$, the formula

$$
\theta_{\phi}(g, h)=\theta(\omega(g, h) \phi) \quad\left(g \in \mathbb{G}(\mathbb{A}), h \in \mathbb{G}^{\prime}(\mathbb{A})\right)
$$

defines a smooth, slowly increasing function on $\mathbb{G}(F) \backslash \mathbb{G}(\mathbb{A}) \times \mathbb{G}^{\prime}(F) \backslash \mathbb{G}^{\prime}(\mathbb{A})$. See $[28,29]$ for these facts.

Let $\sigma=\otimes \sigma_{v}$ be a cuspidal automorphic representation realized on a subspace $H_{\sigma} \subset L^{2}\left(\mathbb{G}^{\prime}(F) \backslash \mathbb{G}^{\prime}(\mathbb{A})\right)$. For each smooth $f \in H_{\sigma}$ the function

$$
\theta_{\phi}^{f}(g)=\int_{\mathbb{G}^{\prime}(F) \backslash \mathbb{G}^{\prime}(\mathbb{A})} \theta_{\phi}(g, h) \overline{f(h)} d h \quad(g \in \mathbb{G}(\mathbb{A}))
$$

is well defined, and determines a slowly increasing function on $\mathbb{G}(F) \backslash \mathbb{G}(\mathbb{A})$. Note, however, that with a bar over $f(h)$ in the integrand, our definition is slightly different from the usual conventions, but is more compatible with local theta correspondence. 
Take $\phi_{1}, \phi_{2} \in S(X(\mathbb{A}))$ and $f_{1}, f_{2} \in H_{\sigma}$, assumed to be smooth. We shall consider the inner product

$$
\left\langle\theta_{\phi_{1}}^{f_{1}}, \theta_{\phi_{2}}^{f_{2}}\right\rangle=\int_{\mathbb{G}(F) \backslash \mathbb{G}(\mathbb{A})} \theta_{\phi_{1}}^{f_{1}}(g) \overline{\theta_{\phi_{2}}^{f_{2}}(g)} d g .
$$

Because of our assumption (4.1), the group $\mathbb{G}$ is anisotropic if and only if $F \neq \mathbb{Q}$. If $\mathbb{G}$ is anisotropic, then the integral in (4.3) is certainly absolutely convergent. If $\mathbb{G}$ is isotropic, then $F=\mathbb{Q}$ and the assumption (4.1) implies that $V$ is of Witt index 1 over $F$. In this case the criterion of [29] again implies that the integral in (4.3) is absolutely convergent.

In any case, the right hand side of (4.3) can be computed via the Rallis inner product formula, which has been worked out in greater generality in [8] using Ichino's extended version of the Siegel-Weil formula for unitary groups [11]. We now explain the formula in the present setting.

In the formalism of L-groups [2, 16], the dual group of $\mathbb{G}$ is ${ }^{L} \mathbb{G}^{0}=\mathrm{GL}(4, \mathbb{C})$, and the non-trivial element of the Galois group $\operatorname{Gal}(E / F)$ acts on $\operatorname{GL}(4, \mathbb{C})$ by an outer automorphism. The semi-direct product $\operatorname{Gal}(E / F) \ltimes \mathrm{GL}(4, \mathbb{C})$ is (a quotient of) the L-group of $\mathbb{G}$ denoted by ${ }^{L} \mathbb{G}$. Now first take the standard representation of GL $(4, \mathbb{C})$ on $\mathbb{C}^{4}$ and then induce it to $\operatorname{Gal}(E / F) \ltimes \mathrm{GL}(4, \mathbb{C})$. This gives an irreducible eightdimensional representation $r$ of the L-group ${ }^{L} \mathbb{G}$. We let $L(s, \sigma)=L(s, \sigma, r)$ be the Langlands L-function attached to the cusp form $\sigma$ and the representation $r$.

Let $\varepsilon=\varepsilon_{E / F}$ be the quadratic character of $C_{F}$ associated to the extension $E / F$, and $L(s, \varepsilon)$ the corresponding Hecke L-function. Let $S_{\infty}$ be the set of Archimedean places of $F$ (all real according to our assumption). Let $S$ be a finite set of places containing $S_{\infty}$, such that $\sigma_{v}$ is unramified for $v \notin S$. Then the partial L-functions

$$
L^{S}(s, \sigma)=\prod_{v \notin S} L_{v}(s, \sigma), \quad L^{S}(s, \varepsilon)=\prod_{v \notin S} L_{v}(s, \varepsilon)
$$

are well defined and entire, so they can be evaluated at any point.

Now suppose the vectors $\phi_{i}, f_{i}$ are all factorizable:

$$
\phi_{i}=\otimes \phi_{i v}, \quad f_{i}=\otimes f_{i v}, \quad i=1,2 .
$$

Assume that for each place $v \notin S$ the following conditions are satisfied:

(a) The groups $\mathbb{G}, \mathbb{G}^{\prime}$ are unramified at $v$.

(b) The vectors $\phi_{i v}, f_{i v}$ are all unramified for $i=1,2$.

(c) One has the usual normalization

$$
\left.\left\langle\phi_{1 v}, \phi_{2 v}\right\rangle=1=f_{1 v}, f_{2 v}\right\rangle .
$$

Then we can express the Rallis inner product formula as

$$
\left\langle\theta_{\phi_{1}}^{f_{1}}, \theta_{\phi_{2}}^{f_{2}}\right\rangle=I_{S}\left(\phi_{1}, \phi_{2} ; f_{1}, f_{2}\right) \cdot \frac{L^{S}\left(\frac{3}{2}, \sigma\right)}{\prod_{k=1}^{4} L^{S}\left(2+k, \varepsilon^{k}\right)},
$$


where

$$
I_{S}\left(\phi_{1}, \phi_{2} ; f_{1}, f_{2}\right)=\prod_{v \in S} I_{v}\left(\phi_{1}, \phi_{2} ; f_{1}, f_{2}\right)
$$

and

$$
I_{v}\left(\phi_{1}, \phi_{2} ; f_{1}, f_{2}\right)=\int_{\mathbb{G}^{\prime}\left(F_{v}\right)}\left\langle\omega_{v}(h) \phi_{1 v}, \phi_{2 v}\right\rangle \overline{\left\langle\sigma_{v}(h) f_{1 v}, f_{2 v}\right\rangle} d h .
$$

We now discuss conditions under which the right hand side of (4.4) will not be identically zero. First observe that if $\sigma$ were one-dimensional then $s=3 / 2$ is precisely the first point where the product $\prod_{v \notin S} L_{v}(s, \sigma)$ fails to converge absolutely. On the other hand if $\sigma$ is infinite dimensional (as will be the case for us) then any non-trivial estimate towards the Ramanujan conjecture on the Hecke eigenvalues imply that the product $\prod_{v \notin S} L_{v}(s, \sigma)$ converges absolutely at $s=3 / 2$. Note that such estimates do exist - for example one could combine base change with the results of [6] or [21]. It follows that $L^{S}\left(\frac{3}{2}, \sigma\right) \neq 0$. Thus it suffices to consider the local integrals in (4.5) for places $v \in S$.

Let $S_{0}$ be the finite set of places where the group $\mathbb{G}$ fails to be quasi-split. By our assumption (4.1) and the conditions for $S$ we have

$$
S_{\infty} \subseteq S_{0} \subseteq S
$$

and a finite place $v$ belongs to $S_{0}$ precisely when the extension $E / F$ does not split at $v$, and that $V$ has Witt index 1 over $F_{v}$. From the local results of Sects. 2 and 3 we can now deduce the following result. Recall that $\mathbb{G}^{\prime}\left(F_{v}\right)$ is isomorphic to either $\mathrm{U}(1,1)$ or $\mathrm{U}(2)$ for any $v \in S_{\infty}$.

\section{Theorem 4.1 Suppose that}

(a) At the place $v_{0}(c f .(4.1)), \sigma_{v}$ is a holomorphic discrete series representation of $\mathbb{G}^{\prime}\left(F_{v_{0}}\right) \simeq \mathrm{U}(1,1)$ that appears in Proposition 2.3 (called $\pi^{\prime}$ there).

(b) For any other $v \in S_{\infty}$, the representation $\sigma_{v}$ is one of the discrete series representations of $\mathbb{G}^{\prime}\left(F_{v}\right)$ discussed in Propositions 2.5.

(c) When $v \in S_{0}$ is finite and $\mathbb{G}^{\prime}\left(F_{v}\right)$ is anisotropic, $\sigma_{v}$ is not the trivial representation.

Then the right hand side of (4.4) is not identically zero. The functions $\theta_{\phi}^{f}$ with $\phi \in S(X(\mathbb{A}))$ and $f \in H_{\sigma}$ generate an irreducible automorphic representation $\pi=$ $\otimes \pi_{v}$ which occurs discretely in $L^{2}(\mathbb{G}(F) \backslash \mathbb{G}(\mathbb{A}))$. In particular, for each $v \in S_{\infty}$, the representation $\pi_{v}$ is the local theta lift of $\sigma_{v}$ described in Proposition 2.3 or 2.5.

Proof We need to make sure that for each $v \in S$ the local integrals (4.5) are not identically zero. Observe that if $v$ is not in $S_{0}$ then the dual pair $\mathbb{G}\left(F_{v}\right), \mathbb{G}^{\prime}\left(F_{v}\right)$ is in the so called stable range, and we obtain the result from Sect. 3 or [18]. If $v$ is in $S_{0}$ and finite then there are two possibilities for $\mathbb{G}^{\prime}\left(F_{v}\right)$. If $\mathbb{G}^{\prime}\left(F_{v}\right)$ is not anisotropic then $\sigma_{v}$ (as a local component of the automorphic cuspidal representation $\sigma$ ) must be infinite dimensional, and in particular non-trivial. So the results of Sect. 3 imply that 
the local integrals (4.5) are not identically zero. Finally if $\mathbb{G}^{\prime}\left(F_{v}\right)$ is anisotropic then our condition (c) and Sect. 3 give what we want.

The assertion about what happens at the Archimedean places follows from the remarks after Proposition 2.3.

If $F \neq \mathbb{Q}$ then $\mathbb{G}$ is anisotropic over $F$, and the automorphic representation $\pi$ is automatically cuspidal. Suppose $F=\mathbb{Q}$. Then our assumption (4.1) implies that $V$ has Witt index 1. Write $V=H \oplus V_{0}$ where $H$ is a hyperbolic plane and $V_{0}$ is anisotropic. Let $\mathbb{G}_{0}=\mathrm{U}\left(V_{0}\right)$. A general principle [22] says that $\pi$ is cuspidal if and only if the theta lift of $\sigma$ to $\mathbb{G}_{0}$ is zero. This last condition can be arranged as follows. Fix a finite place $v_{1}$ where the extension $E / F$ does not split (we could choose $v_{1} \in S_{0}$ ). Let $\pi_{v_{1}}^{\prime}$ be a supercuspidal representation of $\mathbb{G}^{\prime}\left(F_{v_{1}}\right)$. Up to equivalence there are exactly two hermitian forms of dimension 2 over $E_{w}$, where $w$ is the place of $E$ lying over $v$. Consequently there are two unitary groups, one of them being $\mathbb{G}_{0}\left(F_{v}\right)$. Now theta dichotomy [7] says that $\pi_{v_{1}}^{\prime}$ has a non-zero theta lift to exactly one (not both) of these two groups. We choose $\pi_{v_{1}}^{\prime}$ so that it does not lift to $\mathbb{G}_{0}\left(F_{v}\right)$. There exists a cuspidal automorphic representation $\sigma$ of $\mathbb{G}^{\prime}$ satisfying the conditions of Theorem 4.1, and such that $\sigma_{v_{1}} \simeq \pi_{v_{1}}^{\prime}$. It is then clear that $\sigma$ does not lift to $\mathbb{G}_{0}$, and hence $\pi$ is cuspidal.

\section{Conclusions}

We are now in a position to prove Theorem 1.1. Let $\mathbb{G}$ be as in Sect. 4. Then

$$
\mathbb{G}_{\infty} \stackrel{\text { def }}{=} \prod_{v \in S_{\infty}} \mathbb{G}\left(F_{v}\right) \simeq \mathrm{U}(3,1) \times \mathrm{U}(4)^{t},
$$

where $t+1=[F: \mathbb{Q}]$ is the degree of $F$. According to Margulis, the projection of an arithmetic subgroup of $\mathbb{G}(F)$ to $\mathrm{U}(3,1)$ is called an arithmetic subgroup of $\mathrm{U}(3,1)$ : this is what we mean by "an arithmetic subgroup of $\mathrm{U}(3,1)$ arising from hermitian forms over CM-fields" in the statement of Theorem 1.1. Similarly, the projection of a congruence subgroup of $\mathbb{G}(F)$ to $U(3,1)$ is called a congruence subgroup of $U(3,1)$. Note that if $\Gamma$ is a congruence subgroup of $\mathrm{U}(3,1)$ then

$$
\operatorname{det}(\Gamma)=\{\operatorname{det}(\gamma) \mid \gamma \in \Gamma\}
$$

is a congruence subgroup of $\mathrm{U}(1)$ and must be finite. Hence $\Gamma \cap \mathrm{SU}(3,1)$ is of finite index in $\Gamma$.

Let $\Gamma$ be an arithmetic subgroup of $\mathrm{U}(3,1)$, of the type defined above. Let $\pi_{v_{0}}$ be an irreducible unitary representation of $\mathrm{U}(3,1)$ with non-zero cohomology.

We first assume that $\pi_{v_{0}}$ is the representation $A_{\mathfrak{q}}(\lambda)$ described by Proposition 2.2 and (2.3)-(2.4). For each $v \in S_{\infty}$, different from $v_{0}$, we let $\pi_{v}$ be the one-dimensional representation of $U(4)$ discussed in Proposition 2.5. Then by the results of Sect. 4, there exists a cuspidal automorphic representation $\pi$ of $\mathbb{G}(\mathbb{A})$ such that (i) its factor at $v_{0}$ is the representation $A_{\mathfrak{q}}\left(\lambda_{0}\right)$ (cf. Proposition 2.3), and (ii) the factors at the other Archimedean places are the $\pi_{v}$ 's already given above. 
Let $\mathbb{A}_{f}$ be the finite adeles of $F$ and let $K_{f}$ be an open compact subgroup of $\mathbb{G}\left(\mathbb{A}_{f}\right)$, such that $\pi$ has a non-zero vector fixed by $K_{f}$. There is a finite set $\left\{x_{1}, \cdots, x_{m}\right\}$ of elements of $\mathbb{G}\left(\mathbb{A}_{f}\right)$ such that

$$
\mathbb{G}(\mathbb{A})=\bigcup_{j=1}^{m} \mathbb{G}(F) \mathbb{G}_{\infty} x_{j} K_{f}
$$

(a disjoint union). Set $K_{j}=x_{j} K_{f} x_{j}^{-1}$ and $K=\cap K_{j}$. Then $K$ is open compact in $\mathbb{G}\left(\mathbb{A}_{f}\right)$. Let $\Gamma_{0}=\mathbb{G}(F) \cap\left(\mathbb{G}_{\infty} \times K\right)$. Then $\mathbb{G}(F) \backslash \mathbb{G}(\mathbb{A}) / K$ is covered by a disjoint union of finite copies of $\Gamma_{0} \backslash \mathbb{G}_{\infty}$, and pull-back of functions respects the action of $\mathbb{G}_{\infty}$. It follows that the representation

$$
\pi_{\infty}^{0}=A_{\mathfrak{q}}\left(\lambda_{0}\right) \otimes\left(\bigotimes_{v \in S_{\infty} \backslash\left\{v_{0}\right\}} \pi_{v}\right)
$$

occurs in $L^{2}\left(\Gamma_{0} \backslash \mathbb{G}_{\infty}\right)$. Replacing $\Gamma_{0}$ by a finite-index subgroup if necessary, we may assume that all elements of $\Gamma_{0}$ has determinant 1 . Then since $\pi_{v}$ is one-dimensional for each $v \in S_{\infty} \backslash\left\{v_{0}\right\}$, the space of $\pi_{\infty}^{0}$ can be identified with a space of functions on $\Gamma_{0} \backslash \mathrm{U}(3,1)$. Thus $A_{\mathfrak{q}}\left(\lambda_{0}\right)$ occurs in $L^{2}\left(\Gamma_{0} \backslash \mathrm{U}(3,1)\right)$. Let $\Gamma_{1}=\Gamma \cap \Gamma_{0}$. Then $\Gamma_{1}$ is of finite index in $\Gamma$ and $\Gamma_{0}$, and $A_{\mathfrak{q}}\left(\lambda_{0}\right)$ occurs in $L^{2}\left(\Gamma_{1} \backslash \mathrm{U}(3,1)\right)$. Finally, since $A_{\mathfrak{q}}(\lambda)=A_{\mathfrak{q}}\left(\lambda_{0}\right) \otimes \operatorname{det}^{r}$ (cf. (2.5)) and elements of $\Gamma_{1}$ have determinant 1, we see that $A_{\mathfrak{q}}(\lambda)$ also occurs in $L^{2}\left(\Gamma_{1} \backslash \mathrm{U}(3,1)\right)$.

We remark that in the above we have tried to handle theta liftings from various possible forms of $\mathrm{U}\left(V^{\prime}\right)$. But for the purpose of proving Theorem 1.1 we could have just assumed from the very beginning that $V^{\prime}$ is split over $F$. Then the $\pi_{v}$ discussed above would be the trivial representation for each $v \in S_{\infty} \backslash\left\{v_{0}\right\}$, and that condition (c) in Theorem 4.1 would be vacuous.

We now return to our proof. From Lemma 2.1 we see that in any other situation $\pi_{v_{0}}$ would be either holomorphic or anti-holomorphic, or in the discrete series (or one-dimensional, which is the trivial case). But for discrete series representations the result is well known and valid for all arithmetic lattices-not just those arising from hermitian forms, see for example [25]. If $\pi_{v_{0}}$ is holomorphic the result is again known $[1,4,13]$. Alternatively it can be proved in the same way as above, by making different choices of the hermitian space $V^{\prime}$, positive definite at the place $v_{0}$. This concludes our discussion.

Acknowledgements J.-S. Li was supported in part by RGC-GRF grant 602410 of HKSAR. B. Sun was supported in part by NSFC Grant 11222101.

\section{References}

1. Anderson, G.: Theta functions and holomorphic differential forms on compact quotients of bounded symmetric domains. Duke Math. J. 50(4), 1137-1170 (1983)

2. Borel, A.: Automorphic L-functions. In: Automorphic Forms, Representations, and L-Functions. Proc. Symp. Pure Math., vol. 33, pp. 27-61. Am. Math. Soc., Providence (1979). Part 2

3. Borel, A., Casselman, W.: $L^{2}$-cohomology of locally symmetric manifolds of finite volume. Duke Math. J. 50(3), 625-647 (1983) 
4. Borel, A., Wallach, N.: Continuous cohomology, Discrete subgroups, and representations of reductive groups. Ann. Math. Stud. 94 (1980)

5. Clozel, L.: On the cohomology of Kottwitz's arithmetic varieties. Duke Math. J. 72, 757-795 (1993)

6. Gelbart, S., Jacquet, H.: A relation between automorphic forms on $\mathrm{GL}_{2}$ and $\mathrm{GL}_{3}$. Proc. Natl. Acad. Sci. USA 73, 3348-3350 (1976)

7. Harris, M., Kudla, S., Sweet, J.: Theta dichotomy for unitary groups. J. Am. Math. Soc. 9(4), 9411004 (1996)

8. Harris, M., Li, J.S., Skinner, C.: The Rallis inner product formula and $p$-adic $L$-functions. In: Cogdell, J., et al. (eds.) Automorphic Representations, L-Functions and Applications: Progress and Prospects, pp. 225-256 (2005)

9. Harris, M., Li, J.-S., Sun, B.: Theta correspondences for close unitary groups. In: Funke, J., Cogdell, J., Rapoprt, M., Yang, T. (eds.) Arithmetic Geometry and Automorphic Forms, Volume in Honor of the 60th Birthday of Stephen S. Kudla. Advanced Lectures in Mathematics, vol. 19, pp. 265-308. International Press and the Higher Education Press of China, Somerville (2011)

10. Howe, R.: $\theta$ series and invariant theory. In: Automorphic Forms, Representations, and L-Functions. Proc. Symp. Pure Math., vol. 33, pp. 275-285. Am. Math. Soc., Providence (1979)

11. Ichino, A.: A regularized Siegel-Weil formula for unitary groups. Math. Z. 247(2), 241-277 (2004)

12. Kashiwara, M., Vergne, M.: On the Segal-Shale-Weil representations and harmonic polynomials. Invent. Math. 44, 1-47 (1978)

13. Kazhdan, D.: Some applications of the Weil representations. J. Anal. Math. 32, 235-248 (1977)

14. Kudla, S.: Splitting metaplectic covers of dual reductive pairs. Isr. J. Math. 87, 361-401 (1994)

15. Kudla, S.S., Sweet, W.J. Jr.: Degenerate principal series representations for $U(n, n)$. Isr. J. Math. 98, 253-306 (1997)

16. Langlands, R.: Problems in the Theory of Automorphic Forms, Lectures in Modern Analysis and Application. Lecture Notes in Math., vol. 170, pp. 18-86. Springer, New York (1970)

17. Langlands, R.: On the Functional Equations Satisfied by Eisenstein Series. Lecture Note in Math., vol. 544. Springer, Berlin (1976)

18. Li, J.-S.: Singular unitary representations of classical groups. Invent. Math. 97, 237-255 (1989)

19. Li, J.-S.: Theta liftings for unitary representations with non-zero cohomology. Duke Math. J. 61, 913937 (1990)

20. Li, J.-S.: Non-vanishing theorems for the cohomology of certain arithmetic quotients. J. Reine Angew. Math. 428, 177-217 (1992)

21. Luo, W., Rudnick, Z., Sarnak, P.: On the generalized Ramanujan conjecture for GL(n), vol. II. Proc. Symp. Pure Math. 66, 301-311 (1999)

22. Rallis, S.: Langlands functoriality and the Weil representation. Am. J. Math. 104, 469-515 (1982)

23. Rapoport, M., Zink, T.: Über die lokale Zetafunktion von Shimuravarietäten, Monodromiefiltration und verschwindende Zyklen in ungleicher Charakteristik. Invent. Math. 68, 21-101 (1982)

24. Rogawski, J.: Automorphic Representations of Unitary Groups in Three Variables. Ann. of Math. Studies, vol. 123. Princeton University Press, Princeton (1990)

25. Savin, G.: Limit multiplicities of cusp forms. Invent. Math. 95, 149-159 (1989)

26. Sun, B., Zhu, C.-B.: Conservation relations for local theta correspondence. arXiv:1204.2969

27. Vogan, D., Zuckerman, G.: Unitary representations with non-zero cohomology. Compos. Math. 53, 51-90 (1984)

28. Weil, A.: Sur certains groupes d'operateurs unitaires. Acta Math. 111, 143-211 (1964)

29. Weil, A.: Sur la formulae de Siegel dans la theorie des groupes classiques. Acta Math. 113, 1-87 (1965) 